\title{
Le propofol pour réaliser une rachianesthésie en position latérale chez les victimes d'une fracture du fémur
}

\author{
[Propofol to facilitate spinal anesthesia in the lateral position in patients with \\ femoral neck fracture]
}

Vincent Minville MD, ${ }^{*}$ Adeline Castel MD, ${ }^{*}$ Karim Asehnoune MD PhD, $†$ Clément Chassery MD, ${ }^{*}$ Jean-Michel Lafosse MD, $\ddagger$ Luc Nguyen MD, ${ }^{*}$ Aline Colombani MD,${ }^{*}$ Olivier Fourcade MD PhD*

Objectif : Le but de cette étude était d'évaluer la faisabilité et l'efficacité du propofol avant la mobilisation en décubitus latéral chez les patients âgés, victimes d'une fracture de l'extrémité supérieure du fémur, pour la réalisation d'une rachianesthésie.

Méthode : Une étude prospective et descriptive a été réalisée chez 79 patients consécutifs de plus de 75 ans ayant une fracture de l'extrémité supérieure du fémur. Le propofol $(0,5$ $\mathrm{mg} \cdot \mathrm{kg}^{-1}$ ) était injecté au départ. Si la perte de conscience n'était pas obtenue (score de Ramsay $\leq 3 / 6$ ), d'autres injections de $0,25 \mathrm{mg} \cdot \mathrm{kg}^{-1}$ étaient administrées jusqu'à l'obtention d'un score de Ramsay de 4 ou 5 . Ensuite, le patient était placé en décubitus latéral, le membre fracturé vers le haut. L'efficacité du propofol a été évaluée sur la grimace, ainsi que sur le souvenir d'une douleur à la mobilisation. Les données hémodynamiques et la saturation en oxygène étaient aussi notées.

Résultats : Une seule injection de propofol a été nécessaire chez 43 patients, deux injections chez 34 patients et trois injections chez deux patients. Aucune grimace n'a été remarquée dans cette étude, et aucun patient n'a eu le souvenir d'une douleur lors de la mobilisation. Aucune désaturation $\left(\mathrm{SpO}_{2}<\right.$ 92\%), ni chute de tension (diminution de la pression artérielle systolique d'au moins 30\%) n'a été observée.

Conclusion: Le propofol est un moyen simple et efficace d'assurer un confort pendant la mobilisation des patients âgés victimes d'une fracture de l'extrémité supérieure du fémur, pour la réalisation d'une anesthésie médullaire.
Purpose: The aim of this study was to assess the feasibility and efficacy of propofol before positioning elderly patients with a femoral neck fracture in the lateral decubitus position, to perform a spinal anesthetic.

Methods: In this prospective and descriptive study, 79 consecutive patients, $>75 \mathrm{yr}$ old, with a femoral neck fracture were included. Propofol, $0.5 \mathrm{mg} \cdot \mathrm{kg}^{-1}$, was administered. If loss of consciousness was not obtained (Ramsay score $\leq 3 / 6$ ), then additional doses of $0.25 \mathrm{mg} \cdot \mathrm{kg}^{-1}$ were given until a Ramsay score of 4 or 5 was attained. Then, the patient was turned to the lateral decubitus position, the fractured side up. The efficacy of propofol was assessed by observing a grimace during positioning and asking the patients if they had recall of pain. Hemodynamic data and oxygen saturation were collected.

Results: Forty-three patients required a single injection, 34 required two injections and only two patients required three injections. No grimace and no recall of pain were recorded during the study. There was no desaturation $\left(\mathrm{SpO}_{2}<92 \%\right)$, and hypotension, defined as a systolic blood pressure decrease $>30 \%$ from baseline, was observed.

Conclusion: Propofol is a simple and efficacious means of providing comfort while positioning elderly patients with a femoral head fracture before performing spinal anesthesia.

Du Département d'anesthésie et de réanimation, CHU Toulouse-Rangueil, ${ }^{*}$ Université Paul Sabatier, Toulouse ; Département d'anesthésie et de réanimation, Hôpital Paul Brousse, $†$ Villejuif ; et le Service de chirurgie orthopédique et de traumatologie, CHU Toulouse-Rangueil, $\ddagger$ Toulouse, France.

Addresser la correspondance à: Dr Vincent Minville, Département d'anesthésie et de réanimation, CHU Toulouse - Rangueil, Université Paul Sabatier, 1, Avenue Jean Poulhès, 31400 Toulouse, France. Téléphone : 335613227 12; Télécopieur : 33561322232 ;

Courriel : vincentminville@yahoo.fr

Accepté pour publication le 7 août 2006.

Révision acceptée le 19 septembre, 2006.

Conflits d'intérêt et sources de financement : Aucun. 


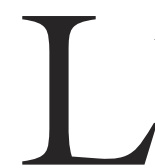

A fracture de l'extrémité supérieure du fémur touche fréquemment les patients âgés, mais elle est grevée d'une forte mortalité. ${ }^{1,2}$ L'anesthésie générale et l'anesthésie locorégionale sont toutes deux associées à des effets secondaires chez ces patients. ${ }^{3-6}$ Cependant, l'anesthésie locorégionale, réalisée sous forme de rachianesthésie, présente certains avantages sur l'anesthésie générale? Des données issues de la littérature montrent qu'une diminution de la dose d'anesthésique local injectée en intrathécal en injection unique ${ }^{8-10}$ ou continue con $^{11,12}$ permet un retentissement hémodynamique moindre.

Néanmoins, les techniques locorégionales nécessitent toutes de mobiliser les patients, soit en décubitus latéral, soit en position assise, afin de permettre la ponction lombaire. Ce positionnement est source de douleur chez ces patients fragiles. ${ }^{13}$ Pour diminuer cette douleur, on a eu recours au bloc du nerf fémoral, qui semble plus efficace que la titration de morphinique $i v{ }^{14}$ Le propofol n'a jamais été évalué pour cette indication, mais a déjà été utilisé chez des sujets âgés pour d'autres interventions. ${ }^{15}$ Le but de cette étude prospective et observationnelle était d'évaluer l'intérêt du propofol chez les patients âgés, atteints d'une fracture de l'extrémité supérieure du fémur, pour la mobilisation en décubitus latéral avant la réalisation d'une rachianesthésie. Les critères de jugement étaient l'absence de réaction à la douleur, la stabilité hémodynamique et l'absence de désaturation.

\section{Patients et méthodes}

Après accord du comité d'éthique du centre hospitalo-universitaire de Toulouse, et après avoir obtenu le consentement éclairé du patient ou de sa famille, tous les patients atteints d'une fracture isolée de l'extrémité supérieure du fémur devant subir une intervention chirurgicale ont été inclus dans une étude prospective et descriptive. Les critères d'exclusion étaient le refus par le malade de l'anesthésie locorégionale, toute autre lésion traumatique associée, un score de Glasgow < 15, une allergie au propofol, des troubles de l'hémostase, une infection au point de ponction lombaire et les troubles du comportement avec agitation. Les patients arrivaient au bloc opératoire après un jeûne d'au moins six heures. Les données démographiques [sexe, age, poids, taille, antécédents, classification du risque selon les critères de l'American Society of Anesthesiologists (ASA)] étaient notées. Après la mise en place d'une voie veineuse périphérique, d'une oxygénothérapie au masque à un débit de $3 \mathrm{~L} \cdot \mathrm{min}^{-1}$ et d'un monitorage standard [électrocardiogramme, pression artérielle non invasive, saturométrie $\left.\left(\mathrm{SpO}_{2}\right)\right]$ les valeurs de base étaient recueillies. Puis un bolus de $0,5 \mathrm{mg} \cdot \mathrm{kg}^{-1}$ de propofol était injecté. Pour l'évaluation de la profondeur de la sédation nous avons utilisé le score de Ramsay: 1) patient anxieux, agité ; 2) patient coopérant, orienté et calme ; 3) patient répondant aux ordres ; 4) patient endormi mais avec une réponse nette à la stimulation de la glabelle ou à un bruit intense ; 5 ) patient endormi répondant faiblement aux stimulations ci-dessus ; et 6) pas de réponse aux stimulations nociceptives. Si la perte de conscience n'était pas obtenue avec la dose initiale (score de Ramsay $\leq 3$ ), d'autres injections de $0,25 \mathrm{mg} \cdot \mathrm{kg}^{-1}$ étaient administrées jusqu'à la perte de conscience (score de Ramsay à 4 ou 5 ). Après la perte de conscience (score de Ramsay à 4 ou 5 ), et après avoir retiré la traction fémorale, les patients étaient placés en décubitus latéral, le membre fracturé vers le haut. L'efficacité du propofol était évaluée sur la grimace visible sur le visage des patients ainsi que sur le souvenir d'une douleur après le réveil (vous souvenez-vous d'un moment douloureux lors de votre mobilisation ?). Aucune autre sédation pouvant altérer la mémoire n'était administrée aux patients. La pression artérielle systolique (PAS), la pression artérielle diastolique (PAD), la fréquence cardiaque (FC) et la $\mathrm{SpO}_{2}$ étaient relevés avant l'injection du propofol (T0), puis à cinq (T5) et à dix (T10) minutes après son injection. Les patients étaient placés en décubitus latéral entre T0 et T5, la rachianesthésie n’était réalisée qu'après $\mathrm{T} 10$ afin de ne pas modifier les paramètres hémodynamiques. L'hypotension était définie par une diminution de la PAS d'au moins $30 \%{ }^{16}$

Les données sont présentées sous forme de moyenne \pm écart-type ou de ratio. Les comparaisons entre les pressions artérielles, la $\mathrm{FC}$ et la $\mathrm{SpO}$ à T0, T5, et T10, ont été réalisées à l'aide d'une ANOVA, puis le test de Mann Whitney (post hoc) a été appliqué. Une valeur de $P<0,05$ a été prise comme seuil de significativité.

\section{Résultats}

Soixante-dix-neuf patients ont été inclus consécutivement dans cette étude. Tous les patients ont été opérés dans les 24 h suivant leur admission à l'hôpital (15h30 $\pm 10 \mathrm{~h}$ ). Les données démographiques et chirurgicales sont présentées dans le Tableau. La dose totale de propofol utilisée était de $37 \pm 19 \mathrm{mg}$ : une seule injection a été nécessaire chez 43 patients, deux injections chez 34 patients et trois injections chez deux patients. Aucun patient n'a grimacé lors du retournement. Aucun patient n'a eu un souvenir d'une douleur lors de la procédure.

Les variations de la pression artérielle sont représentées dans la Figure. La PAS et la PAD ont diminué ( $P$ 
TABLEAU Données démographiques et chirurgicales

\begin{tabular}{ll}
\hline & $n=79$ \\
\hline Age (années) & $86 \pm 5$ \\
Poids (kg) & $60 \pm 12$ \\
Taille (cm) & $160 \pm 8$ \\
ASA I/II/III/IV & $7 / 41 / 29 / 2$ \\
Type d'anesthésie (RA/RAC) & $29 / 50$ \\
Sexe (F/M) & $64 / 15$ \\
ATCD & $4 / 8 / 28 / 11 / 12 / 5 / 3$ \\
(AC/IC/HTA/C/D/BPCO/AVC) & $43 / 26 / 10$ \\
Type de chirurgie (DHS/PdM/PTH) & $63 \pm 21$ \\
Durée de la chirurgie (min) & \\
\hline Les données sont exprimées en moyenne \pm écart type ou ratio. \\
ASA = Classification de l'American Society of Anesthsiologists ; \\
RA = rachianesthésie ; RAC = rachianesthésie continue ; ATCD \\
= antécédent ; AC = arythmie cardiaque ; IC = insuffisance cardi- \\
aque ; HTA = hypertension artérielle ; C = maladie coronarienne ; \\
D = diabète ; BPCO = broncho-pneumopathie chronique obstruc- \\
tive ; AVC = accident vasculaire cérébral ; DHS = clou et plaque ; \\
PdM = prothèse de Moore ; PTH = prothèse totale de hanche.
\end{tabular}

$<0,05)$ dans les cinq premières minutes, mais pas de cinq à dix minutes après l'injection du propofol. Il n'y a eu aucune hypotension (i.e., diminution $>30$ $\%$ de la valeur initiale), et aucun patient n'a eu besoin d'éphédrine. La FC n'a pas varié de façon significative (T0 : = $81 \pm 13 \mathrm{~min}^{-1}$, T5 : $80 \pm 11 \mathrm{~min}^{-1}$ et Tl0: 79 $\left.\pm 10 \mathrm{~min}^{-1}\right)$, ni la $\mathrm{SpO}_{2}(\mathrm{~T} 0: 97 \pm 2 \%$, T5 : $98 \pm 2 \%$ et T10 : $=98 \pm 2 \%)$. Aucun patient n'a dû être ventilé au masque facial. Aucun incident ni aucune complication n'ont été observés lors de cette étude.

\section{Discussion}

Dans cette étude prospective, nous avons démontré que le propofol permettait de mobiliser en décubitus latéral les patients âgés de plus de 75 ans, victimes d'une fracture de l'extrémité supérieure du fémur, pour bénéficier d'une rachianesthésie de manière confortable, sans manifestation de douleur et sans retentissement hémodynamique cliniquement significatif.

La seule étude se rapportant à ce sujet est celle de Sia et coll. ${ }^{14}$ Les auteurs concluaient que le bloc fémoral était plus efficace qu'une titration iv de fentanyl (bolus itératifs de $3 \mu \mathrm{g} \cdot \mathrm{kg}^{-1}$ ). ${ }^{14}$ Cependant cette étude concernait des sujets jeunes victimes d'une fracture de la diaphyse fémorale. En outre, ils pratiquaient la rachianesthésie en position assise, ce qui nous semble difficilement réalisable chez des sujets âgés. Dans notre établissement, l'anesthésie médullaire est réalisée en décubitus latéral avec le membre fracturé vers le haut. En effet, nous utilisons de la bupivacaïne isobare et les patients sont laissés en position latérale cinq minutes afin de fixer l'anesthésie pour pouvoir

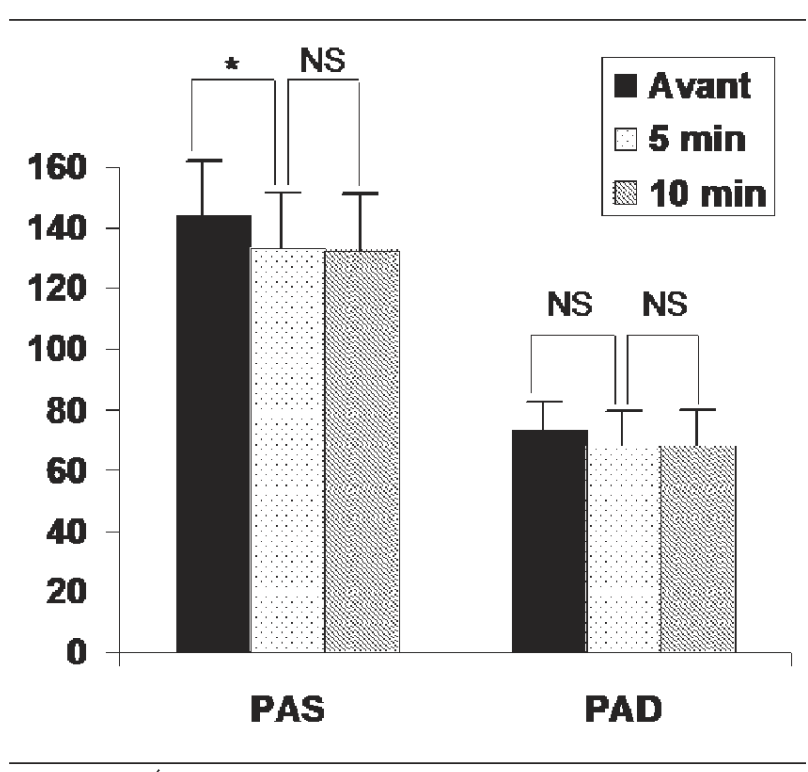

FIGURE Évolution de la pression artérielle systolique (PAS) et diastolique (PAD), en $\mathrm{mmHg}$, avant l'injection de propofol, à cinq et à dix minutes après l'injection de propofol. ${ }^{*} P<0,05 ;$ NS $=$ non significatif.

les remettre en décubitus dorsal sans douleur. Cette procédure a déjà été décrite par d'autres auteurs qui réalisaient une rachianesthésie hypobare unilatérale. ${ }^{10}$

Nous préférons le propofol à la titration morphinique, qui est efficace, ${ }^{17}$ et utilisable facilement chez le sujet âgé, ${ }^{18}$ mais nécessite plus de temps. De plus le propofol est plus adapté que le midazolam pour la sédation courte, et ce même chez les patients âgés. ${ }^{15}$ L'hypotension était définie par une diminution de la PAS d'au moins $30 \%{ }^{16}$ Aucun patient n'a atteint ce critère. Un autre avantage du propofol est son effet anti-nauséeux ainsi que son confort plus important que le midazolam pour les patients. ${ }^{19}$ En outre, l'utilisation des morphiniques augmente le risque de détresse respiratoire dans les 24 h postopératoires. ${ }^{20}$

Une limitation de notre étude est son caractère descriptif. Cependant, les résultats sont assez clairs et portent sur un nombre suffisant de patients pour pouvoir être utilisés en pratique courante. Le propofol a aussi été utilisé, avec de bons résultats, pour des sédations courtes chez les sujets âgés dans d'autres indications. ${ }^{15}$ Une autre limite de cette étude est l'évaluation objective mais non quantitative de la douleur. Effectivement, les études concernant l'analgésie utilisent le plus souvent l'échelle visuelle analogique. Toutefois, chez les sujets âgés, sa compréhension et 
son interprétation sont souvent difficiles. ${ }^{21}$ Ainsi, nous avons choisi l'apparition d'une grimace lors du retournement, et la mémorisation de la douleur du patient, qui nous semblaient être les moyens les plus objectifs pour évaluer cette douleur.

L'analyse de cette série démontre donc l'intérêt d'une perte de conscience rapide et brève, induite par le propofol, pour la mobilisation en décubitus latéral des patients de plus de 75 ans avant une anesthésie médullaire. Les faibles doses utilisées permettent d'obtenir une sédation suffisante sans effets délétères hémodynamiques ni respiratoires. Cette gestion simple et efficace de la douleur et du stress périopératoire pourrait contribuer à la réhabilitation globale du sujet âgé après fracture de l'extrémité supérieure du fémur.

\section{Références}

1 Roberts SE, Goldacre MJ. Time trends and demography of mortality after fractured neck of femur in an English population, 1968-98: database study. BMJ 2003; 327: $771-5$.

2 Rosencher $\mathrm{N}$, Vielpeau , Emmerich, Fagnani 1 , Samama CM; the ESCORTE Group. Venous thromboembolism and mortality after hip fracture surgery: the ESCORTE study. J Thromb Haemost 2005; 3 : 2006-14.

3 Berggren D, Gustafson $\Upsilon$, Eriksson B, et al. Postoperative confusion after anesthesia in elderly patients with femoral neck fractures. Anesth Analg 1987; 66: 497-504.

4 Reilly CS. Regional analgesia and myocardial ischaemia. Br J Anaesth 1993; 71: 467-8.

5 McKenzie PJ, Wishart HY, Dewar KM, Gray I, Smith $G$. Comparison of the effects of spinal anaesthesia and general anaesthesia on postoperative oxygenation and perioperative mortality. Br J Anaesth 1980; 52: 49-54.

6 Reich DI, Hossain \$ Krol M, et al. Predictors of hypotension after induction of general anesthesia. Anesth Analg. 2005; 101: 622-8.

7 Brown A, Visram A, Jones R, Irwin MG, Bacon-Shone $J$. Preoperative and postoperative oxygen saturation in the elderly following spinal or general anesthesia - an audit of current practice. Anaesth Intensive Care 1994; 22: 150-4.

8 Ben-David B, Frankel R, Arzumonov T, Marchevsky $\Upsilon$, Volpin $G$. Minidose bupivacaine-fentanyl spinal anesthesia for surgical repair of hip fracture in the aged. Anesthesiology 2000; 92: 6-10.

9 Asehnoune K, Larousse E, Tadie JM, Minville V, Droupy $S$, Benhamou D. Small-dose bupivacaine-sufentanil prevents cardiac output modifications after spinal anesthesia. Anesth Analg 2005; 101: 1512-5.

10 Khatouf M, Loughnane F, Boini S, et al. Unilateral spinal anaesthesia in elderly patients for hip trauma: a pilot study (French). Ann Fr Anesth Réanim 2005; 24: 249-54.

11 Favarel-Garrigues JF, Sztark F, Petitjean ME, Thicoipe $M$, Lassie P, Dabadie P. Hemodynamic effects of spinal anesthesia in the elderly: single dose versus titration through a catheter. Anesth Analg 1996; 82: 312-6.

12 Minville V, Fourcade O, Grousset D, et al. Spinal anesthesia using single injection low-dose bupivacaine versus continuous catheter injection techniques for surgical repair of hip fracture in elderly patients. Anesth Analg 2006; 102: 1559-63.

13 Halm EA, Magaziner, Hannan EL, et al. Frequency and impact of active clinical issues and new impairments on hospital discharge in patients with hip fracture. Arch Intern Med 2003; 163: 108-13.

14 Sia S, Pelusio F, Barbagli R, Rivituso C. Analgesia before performing a spinal block in the sitting position in patients with femoral shaft fracture: a comparison between femoral nerve block and intravenous fentanyl. Anesth Analg 2004; 99: 1221-4.

15 Riphaus A, Stergiou N, Webrmann T. Sedation with propofol for routine ERCP in high-risk octogenarians: a randomized, controlled study. Am J Gastroenterol 2005; 100: 1957-63.

16 Hartmann B, Junger A, Klasen J, et al. The incidence and risk factors for hypotension after spinal anesthesia induction: an analysis with automated data collection. Anesth Analg 2002; 94: 1521-9.

17 Aubrun B, Valade $\mathrm{A}$, Riou B. Intravenous morphine titration (French). Ann Fr Anesth Reanim 2004; 23: 973-85.

18 Aubrun P, Bunge D, Langeron D, Saillant G, Coriat P, Riou B. Postoperative morphine consumption in the elderly patient. Anesthesiology 2003; 99: 160-5.

19 Dikmen Mentes 3, Unsal D, Baran O, Argun G, Ertunc $F N$. Effect of sedation with midazolam or propofol on patient's comfort during cancer chemotherapy infusion: a prospective, randomized, double-blind study in breast cancer patients. J Chemother 2005; 17: 327-33.

20 Taylor S, Kirton OQ, StaffA, Kozol RA. Postoperative day one: a high risk period for respiratory events. Am J Surg 2005; 190: 752-6.

21 Gagliese 1, Weizblit N, Ellis W, Chan VW. The measurement of postoperative pain: a comparison of intensity scales in younger and older surgical patients. Pain 2005; 117: 412-20. 